\title{
Scheugenpflug Technologietage 2013 in neuer Fertigungshalle
}

Insgesamt 300 Fachleute aus 20 Nationen folgten der Einladung der Scheugenpflug AG zu den diesjährigen Technologietagen, die Ende April in Neustadt an der Donau veranstaltet wurden. Neben zehn Fachvorträgen wurden praxisnah Produktinnovationen aus den Bereichen Vakuumtechnologie, Entgasung von Dosiermedien und der Prozessautomatisierung im neuen $6.000 \mathrm{~m}^{2}$ großen Fertigungsbereich präsentiert.

$D_{d}$ Teilnahme von acht Vergussmedienherstellern und zwei Anlagenbauern als Referenten und Aussteller unterstreicht einmal mehr die Bedeutung der Abstimmung von Vergussmedium und Dosiertechnologie. „Eine einmalige Gelegenheit, neue und modernste Dosiertechnologie auf engstem Raum live zu erleben“, so Steffen Köhler von ml\&s GmbH \& Co. KG, einem KomplettDienstleister für die Elektronikbranche aus Greifswald.

Insbesondere das neue Prozessmodul stand im Fokus der Fachbesucher. Mit diesem Kompaktsystem ist es nach Herstellerangaben möglich, die neueste Dosiertechnologie in jede Fertigungslinie einfach und sicher zu integrieren. Die für die Vergussqualität wichtigen Komponenten Dosierkopf, automatische Nadelvermessung, Topfzeitkontrolle und Becher-Wiegeposition sind über das vorinstallierte Achsensystem und die zugehörige Steuerung zu einer geprüften Einheit verbunden. Externer Programmieraufwand entfällt. Das aufeinander abgestimmte Kompaktsystem garantiert eine schnelle Integrati-

\section{Ardex wächst weltweit}

U m seine strategische Position in Ozeanien zu stärken, hat das Unternehmen Ardex, Hersteller bauchemischer Materialien, die Mehrheit an Cemix in Neuseeland, einem Anbieter in den Bereichen Trockenmörtel und Belagsverlegung, übernommen.

Mit Cemix besitzt die Ardex Gruppe nun die zweite Gesellschaft in Neuseeland. Ardex Neuseeland mit Sitz in Christchurch ist in dieser Region be-

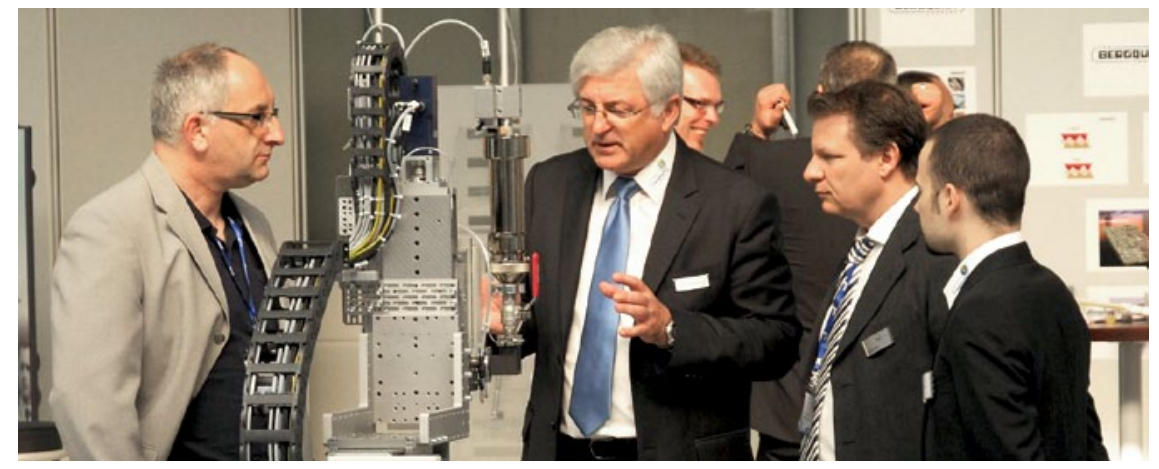

Das Prozessmodul stand im Fokus der Fachbesucher. Firmengründer Erich Scheugenpflug (Mitte) erläutert die Vorteile dieses Kompaktsystems bei der Integration in eine Fertigungslinie.

on und die optimale Vergussqualität von Beginn an.

Weitere Highlights der Technologietage waren die immer wichtiger werdenden Optionen zur wirkungsvollen Entgasung von Hochleistungsvergussmedien und der letzte Stand der Vakuumvergusstechnologie. Totraum- und luftblasenfreier Verguss ist ein wichtiger Garant für einwandfreie Bauteilfunktionalität und lange Lebensdauer. Modernste Dünnschichtentgasungstechnologie und die blasenfreie Materialbereitstellung über den gesamten Dosierprozess standen hier im Zentrum des Interesses.

„Die hohe fachliche Kompetenz der Besucher aus den Bereichen Prozesstechnik und Entwicklung haben mich sehr beeindruckt“, so Holger Schuh von The Bergquist Company, einer der Referenten und Aussteller bei den Scheugenpflug Technologietagen 2013. „Die praxisnah gezeigte Kombination aus Vergussmaterial und -technologie spiegelt die wichtigen Parameter für eine hohe Bauteilqualität wieder.“ reits seit Jahren als Anbieter von Abdichtungsprodukten aktiv. Beide Gesellschaften werden in Zukunft den Markt mit ihrem jeweiligen individuellen Marken- und Leistungsprofilen parallel bearbeiten; dennoch werden sich Synergien in den Bereichen Produktion, Vertrieb und Einkauf ergeben.

Gegenwärtig sind bei Ardex weltweit insgesamt 2.010 Mitarbeiter beschäftigt. Es bestehen Produktions- so- wie Vertriebsgesellschaften in Australien, Benelux, Bulgarien, China, Dänemark, Deutschland, Dubai, Finnland, Frankreich, Großbritannien, Hongkong, Indien, Irland, Italien, Kanada, Korea, Kroatien, Malaysia, Mexiko, Neuseeland, Norwegen, Österreich, Polen, Rumänien, Russland, Schweden, Schweiz, Singapur, Spanien, Taiwan, Tschechien, Türkei, Ungarn, den USA und Vietnam. 